\title{
Aggregating preference rankings using an optimistic-pessimistic approach: Closed-form expressions
}

\author{
Bonifacio Llamazares* \\ Departamento de Economía Aplicada, Instituto de Matemáticas (IMUVA), and BORDA Research Unit, Universidad de \\ Valladolid, Avda. Valle de Esgueva 6, 47011 Valladolid, Spain.
}

\begin{abstract}
There exist in the literature several models to tackle the problem of aggregating preferences rankings where each alternative is evaluated with the most favorable scoring vector for it (which can be considered as an optimistic approach). Recently, Khodabakhshi and Aryavash [M. Khodabakhshi, K. Aryavash, Aggregating preference rankings using an optimistic-pessimistic approach, Computers \& Industrial Engineering 85 (2015) 13-16] have suggested a new model where both the optimistic and the pessimistic approaches are taken into account. In this paper we provide closed-form expressions for the scores of alternatives when the model proposed by these authors is used. The expressions obtained allow us to analyze the model and suggest some small modifications.

Keywords: Khodabakhshi and Aryavash's model, Aggregating preference rankings, Scoring rules, Data Envelopment Analysis (DEA).
\end{abstract}

\section{Introduction}

The problem of obtaining a collective ranking from a set of individual rankings has been extensively studied by many authors, specially in some areas such as Social Choice Theory and Operations Research (it is worth noting that this problem can be seen as a specific case of a multi-criteria decision making problem where all the weights are equal). Among the wide variety of procedures existing in the literature (see Chebotarev and Shamis, 1998, for a review of scoring methods), scoring rules play a major role due to their simplicity and properties (see, for instance, Merlin, 2003; Llamazares and Peña, 2015b).

Each scoring rule is associated with a scoring vector $\left(w_{1}, \ldots, w_{m}\right)$, so each alternative receives $w_{1}$ points each time it is ranked first, $w_{2}$ points each time it is ranked second, and so on; and alternatives are ordered

\footnotetext{
*Corresponding author. Tel.: +34-983-186544; fax: +34-983-423299.

Email address: boni@eco.uva.es (Bonifacio Llamazares)
} 
according to the total number of points they receive. Evidently, a crucial issue in this framework is the choice of the scoring vector, since, as it is well known, the ordering of alternatives may depend on the scoring vector used. It is worth mentioning that in addition to the numerous academic examples found in the literature (see, for instance, Fishburn, 1981), Llamazares and Peña (2013) and Llamazares (2016) have also shown this fact through scoring vectors used in some sports competitions (concretely, in the Formula One World Championship and in the Motorcycle World Championship).

To avoid a subjective choice of the scoring vector, Cook and Kress (1990) proposed a model based on Data Envelopment Analysis (DEA) to assess each alternative with the most favorable scoring vector for it. Nevertheless, as it is usual in the DEA methodology, various alternatives are often efficient, that is, they achieve the maximum score. For this reason, different models have been proposed in the literature to discriminate among efficient alternatives (see, for instance, Green et al., 1996; Hashimoto, 1997; Obata and Ishii, 2003; Foroughi et al., 2005; Foroughi and Tamiz, 2005; Wang and Chin, 2007; Wang et al., 2008). An analysis of some of them can be found in Llamazares and Peña (2009).

Besides the above models, which are based on DEA methodology, there exist other similar models where variable weights are also used (see, for instance, Hashimoto and Wu (2004); Contreras et al. (2005); Wang et al. (2007a,b); Contreras (2011); Ebrahimnejad (2012); Foroughi and Aouni (2012); Hosseinzadeh Lotfi et al. (2013); Llamazares and Peña (2013); Hadi-Vencheh (2014); Khodabakhshi and Aryavash (2015); Llamazares (2016)).

Among this great variety of models, in this paper we focus on the approach proposed by Khodabakhshi and Aryavash (2015). These authors consider both the optimistic and pessimistic approaches, for which they maximize and minimize, respectively, the scores of alternatives by considering several restrictions on the weights. Namely, they are a strictly decreasing sequence of values greater than zero and their sum has to be equal to one divided by the number of voters. Once the minimum and maximum values of the scores are obtained, it is necessary to get a single value that represents the score of each alternative. To do this, these authors consider convex combinations of the minimum and maximum values of the scores by using the same parameter $\lambda \in(0,1)$ for all of them, and impose that the sum of the overall scores has to be equal to one.

In this paper we provide closed-form expressions for the minimum and maximum scores obtained by the alternatives when the model proposed by these authors is used. The knowledge of these expressions has a double advantage. On the one hand, it is possible to rank the alternatives without the need to solve the 
proposed model. On the other hand, they also allow us to analyze more deeply some features of the model proposed by these authors. Following this analysis, some modifications are suggested to improve model performance.

The rest of the paper is organized as follows. In Section 2 we recall Khodabakhshi and Aryavash's model. Section 3 is devoted to provide closed-form expressions for the minimum and maximum scores assigned to the alternatives with Khodabakhshi and Aryavash's model. In Section 4 we use the expressions obtained to carry out an analysis of some features of the model. Finally, some concluding remarks are provided in Section 5.

\section{Khodabakhshi and Aryavash's model}

Let $\mathcal{A}=\left\{A_{1}, \ldots, A_{n}\right\}$ be a set of alternatives and suppose that each voter of a set of $k$ voters selects $m$ alternatives and ranks them from the most to the least preferred. Under the scoring rule associated with the scoring vector $\left(w_{1}, \ldots, w_{m}\right)$, the alternative $A_{j}$ receives $s_{j}=\sum_{i=1}^{m} x_{i j} w_{i}$ points, where $x_{i j}(i=1, \ldots, n)$ is the number of $i$ th place votes of alternative $A_{j}$ and $w_{i}$ represents the weight of rank $i$.

Models based on DEA methodology usually propose to evaluate each alternative with the most favorable scoring vector for him/her by assuming certain constraints on the weights. In the case of the model proposed by Khodabakhshi and Aryavash, the normalization assumption $\sum_{j=1}^{n} s_{j}=1$ is imposed. From this condition, the authors obtain the restriction

$$
\sum_{i=1}^{m} w_{i}=\frac{1}{k}
$$

Moreover, since the $i$ th place of ranking is preferred to the $(i+1)$ th place, the weights should satisfy $w_{1}>w_{2}>\cdots>w_{m}$. Therefore, they also consider the constraints $w_{i}-w_{i+1}>0(i=1, \ldots, m-1)$. Likewise, they add the constraint $w_{m}>0$ to avoid the appearance of zero weights.

The main difference between the model proposed by these authors and others existing in the literature is that they suggest to determine the scores of alternatives by using both pessimistic and optimistic approaches. To do this, the minimum and maximum scores of each alternative $A_{o}$ is calculated by means of the following model: 


$$
\begin{aligned}
\min \text { and } \max & s_{o}=\sum_{i=1}^{m} x_{i o} w_{i}, \\
\text { s.t. } & \sum_{i=1}^{m} w_{i}=\frac{1}{k} \\
& w_{i}-w_{i+1}>0, \quad i=1, \ldots, m-1, \\
& w_{m}>0,
\end{aligned}
$$

Given that the feasible set of Model (1) is not closed, there exist the infimum and supremum $\left(s_{o}^{\inf }\right.$ and $s_{o}^{\text {sup }}$ ), but not the minimum and maximum values of Model (1). For this reason, the authors suggest to solve the following model,

$$
\begin{aligned}
\min \text { and } \max & \bar{s}_{o}=\sum_{i=1}^{m} x_{i o} w_{i}, \\
\text { s.t. } & \sum_{i=1}^{m} w_{i}=\frac{1}{k} \\
& w_{i}-w_{i+1} \geq 0, \quad i=1, \ldots, m-1, \\
& w_{m} \geq 0,
\end{aligned}
$$

taking into account that the minimum value, $\bar{s}_{o}^{\text {min }}$, coincides with $s_{o}^{\text {inf }}$ whereas the maximum value, $\bar{s}_{o}^{\text {max }}$, coincides with $s_{o}^{\text {sup }}$.

Notice that when we consider the feasible set of Model (1) the values $s_{o}=\sum_{i=1}^{m} x_{i o} w_{i}$ are located in the interval $\left(s_{o}^{\text {inf }}, s_{o}^{\text {sup }}\right)=\left(\bar{s}_{o}^{\min }, \bar{s}_{o}^{\max }\right)$. To obtain a single value that represents the score of alternative $A_{o}$, these authors propose the following method. Given that

$$
s_{j}^{\text {inf }}<s_{j}<s_{j}^{\text {sup }}, \quad j=1, \ldots, n,
$$

there exist parameters $\lambda_{j} \in(0,1)(j=1, \ldots, n)$ such that

$$
s_{j}=s_{j}^{\text {inf }} \lambda_{j}+s_{j}^{\text {sup }}\left(1-\lambda_{j}\right), \quad j=1, \ldots, n .
$$

To get the scores of alternatives in a fair way, all parameters $\lambda_{j}$ should be equal. To do this, these authors consider the normalization assumption $\sum_{j=1}^{n} s_{j}=1$. So, the values $s_{j}$ can be determined by solving the following linear equations system:

$$
\left\{\begin{array}{l}
s_{j}=s_{j}^{\text {inf }} \lambda+s_{j}^{\text {sup }}(1-\lambda), \quad j=1, \ldots, n, \\
\sum_{j=1}^{n} s_{j}=1 .
\end{array}\right.
$$


The scores obtained are denoted by $s_{j}^{M}$ and the alternatives are ordered according to these scores. If two scores are equal, the tie is broken by using the lexicographic order of the vectors $\left(x_{1 j}, \ldots, x_{m j}\right)$. In Algorithm 1 we show the algorithm given by Khodabakhshi and Aryavash for ranking the alternatives $A_{p}$ and $A_{q}$ (notice that rank $A_{p}$ denotes the position of this alternative in the overall order. Therefore, when alternative $A_{p}$ has a better score than $A_{q}$ we write $\operatorname{rank} A_{p}<\operatorname{rank} A_{q}$ ).

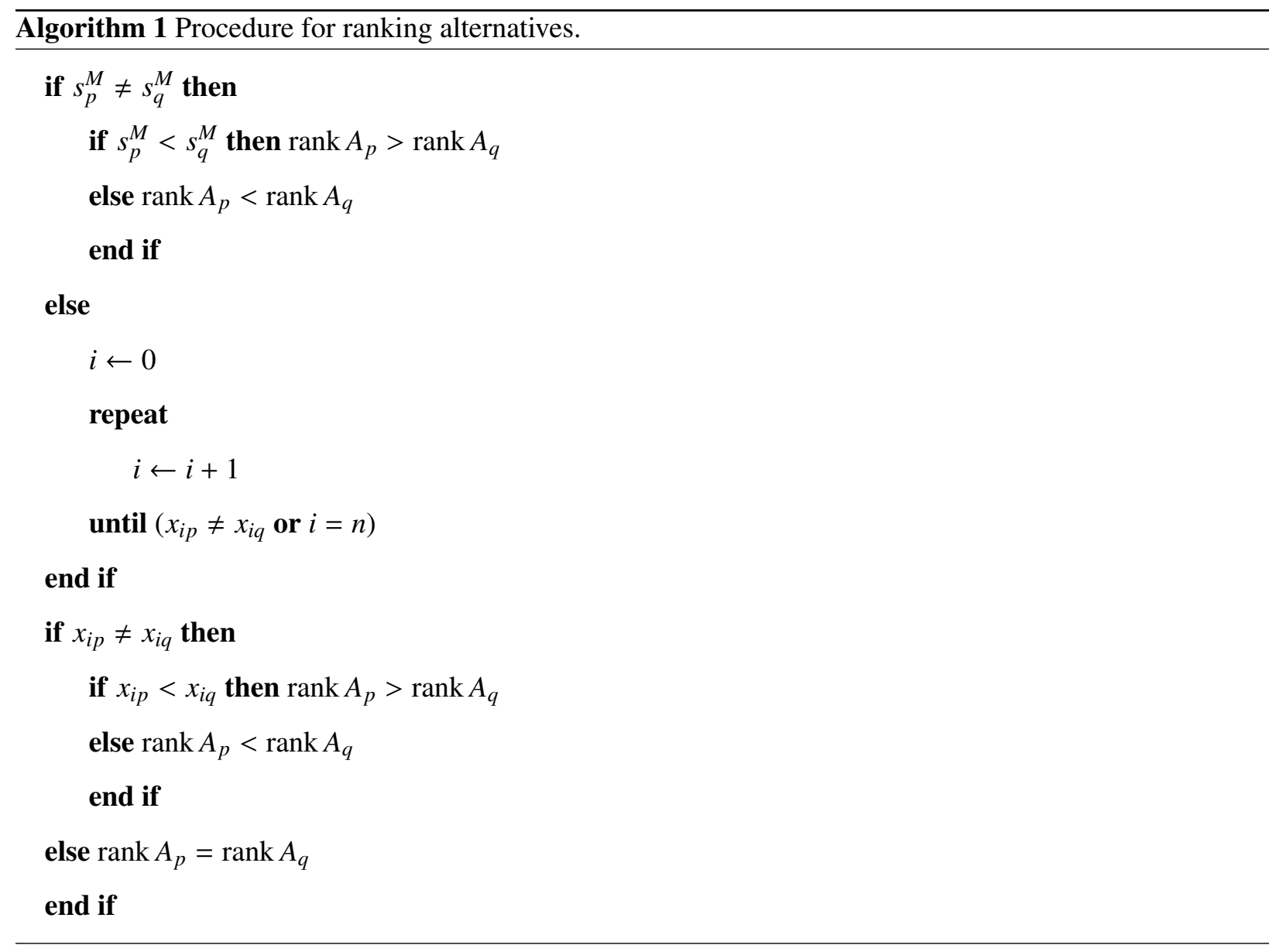

\section{Closed-form expressions for Khodabakhshi and Aryavash's model}

In this section we provide closed-form expressions for the model proposed by Khodabakhshi and Aryavash. Firstly, we give an alternative representation of the model where we replace the constraints $w_{i}-w_{i+1}>0(i=1, \ldots, m-1)$ by nonnegativity of the variables. 
Proposition 1. Model (2) can be expressed as

$$
\begin{aligned}
& \min \text { and } \max \quad \bar{s}_{o}=\sum_{i=1}^{m} X_{i o} W_{i}, \\
& \text { s.t. } \sum_{i=1}^{m} i W_{i}=\frac{1}{k} \\
& W_{i} \geq 0, \quad i=1, \ldots, m,
\end{aligned}
$$

where $X_{i o}=\sum_{j=1}^{i} x_{j o}$ for all $i \in\{1, \ldots, m\}$.

Proof. In Model (2) we make the following change of variables:

$$
\left\{\begin{array}{l}
W_{i}=w_{i}-w_{i+1}, \quad i=1, \ldots, m-1, \\
W_{m}=w_{m} .
\end{array}\right.
$$

It is easy to check that $w_{i}=\sum_{j=i}^{m} W_{j}$ for all $i \in\{1, \ldots, m\}$. Moreover,

$$
\begin{aligned}
\sum_{i=1}^{m} x_{i o} w_{i} & =\sum_{i=1}^{m} x_{i o} \sum_{j=i}^{m} W_{j}=\sum_{\substack{1 \leq i \leq m \\
i \leq j \leq m}} x_{i o} W_{j}=\sum_{1 \leq i \leq j \leq m} x_{i o} W_{j}=\sum_{\substack{1 \leq j \leq m \\
1 \leq i \leq j}} x_{i o} W_{j}=\sum_{j=1}^{m} \sum_{i=1}^{j} x_{i o} W_{j} \\
& =\sum_{j=1}^{m} W_{j} \sum_{i=1}^{j} x_{i o}=\sum_{i=1}^{m} W_{i} \sum_{j=1}^{i} x_{j o}
\end{aligned}
$$

where the last equality is got by switching the role of $i$ and $j$. In a similar way we can obtain

$$
\sum_{i=1}^{m} w_{i}=\sum_{i=1}^{m} i W_{i}
$$

The result follows by using the notation $X_{i o}=\sum_{j=1}^{i} x_{j o}$.

It is worth mentioning that the values $X_{i o}=\sum_{j=1}^{i} x_{j o}$ are called cumulative standing and play an important role in many models built from scoring rules (see, for instance, Fishburn, 1974; Moulin, 1988, cap. 9; Stein et al., 1994; Green et al., 1996; Llamazares and Peña, 2009, 2013, 2015a,b).

Next we give the optimal solution of Model (4) for both the pessimistic and optimistic approaches.

Theorem 1. Consider Model (4). Then

$$
\begin{aligned}
& \bar{s}_{o}^{\min }=\frac{1}{k} \min _{i=1, \ldots, m} \frac{X_{i o}}{i}, \\
& \bar{s}_{o}^{\text {max }}=\frac{1}{k} \max _{i=1, \ldots, m} \frac{X_{i o}}{i} .
\end{aligned}
$$


Proof. It is well know that if a linear program has a finite optimal solution, it has an optimal extreme point solution (see, for instance, Bazaraa et al., 2009). The result follows from the fact that the extreme points of the feasible set of Model (4) are $1 /(k i) \mathbf{e}_{i}$, where $\mathbf{e}_{i}$ denotes the vector with 1 in the $i$ th coordinate and 0 elsewhere.

The knowledge of the closed-form expressions of the infimum and supremum scores of alternatives allows us their calculation without solving Model (2). Moreover, it can be carried out easily through a spreadsheet or by means of Algorithm 2.

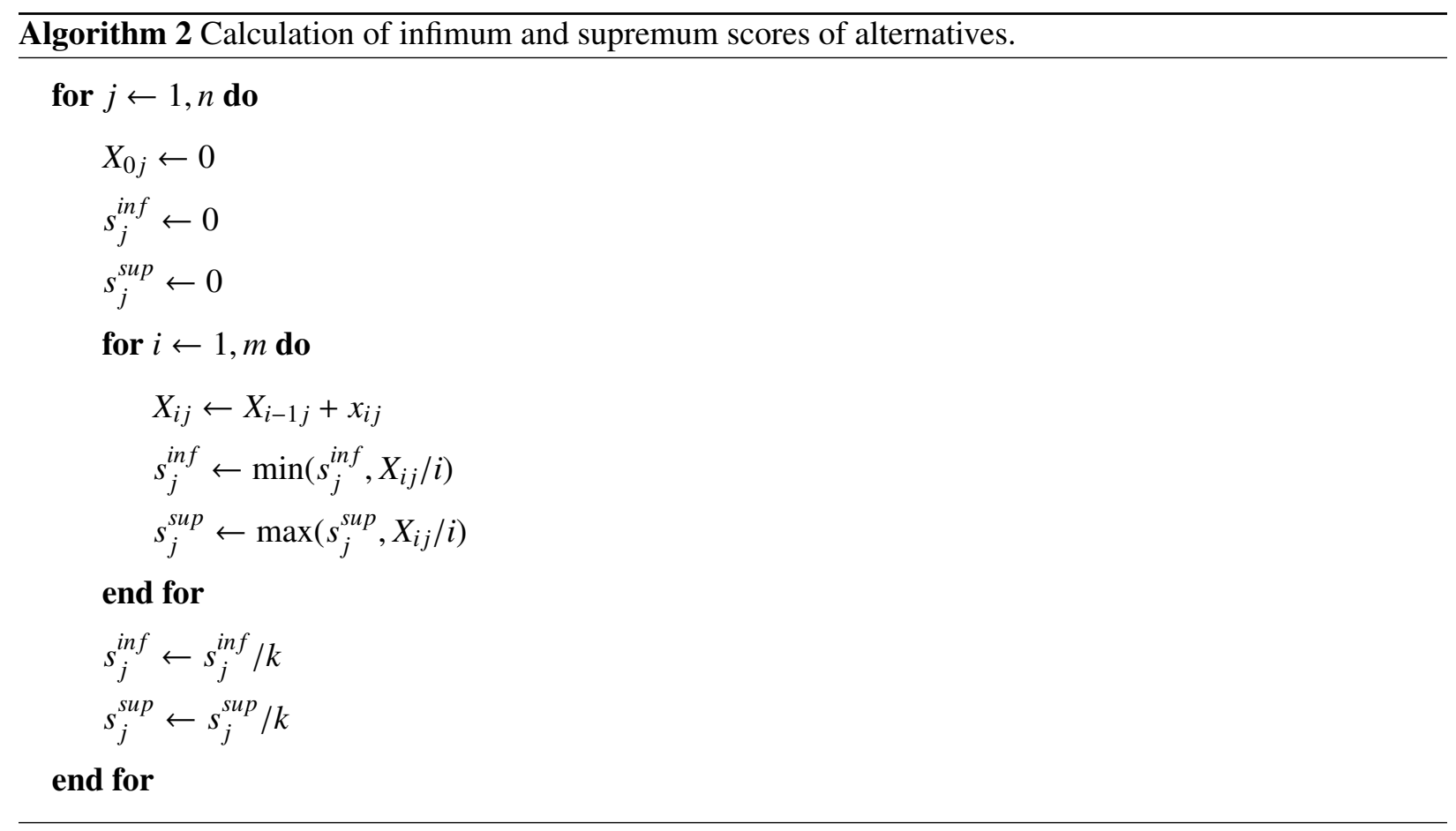

Once we have obtained the infimum and supremum scores of alternatives, the value of $\lambda$ in System (3) can be easily obtained by means of the expression

$$
\lambda=\frac{\sum_{j=1}^{n} s_{j}^{\text {sup }}-1}{\sum_{j=1}^{n} s_{j}^{\text {sup }}-\sum_{j=1}^{n} s_{j}^{\text {inf }}},
$$

and the global score of each alternative is given by

$$
s_{j}^{M}=s_{j}^{\text {inf }} \lambda+s_{j}^{\text {sup }}(1-\lambda), \quad j=1, \ldots, n .
$$




\section{Analysis of Khodabakhshi and Aryavash's model}

The knowledge of the closed-form expressions that represent the infimum and supremum scores of alternatives allows us to analyze some features of the model. For instance, by Theorem 1 we know that

$$
\begin{aligned}
& s_{j}^{\text {inf }}=\frac{1}{k} \min _{i=1, \ldots, m} \frac{X_{i j}}{i}, \\
& s_{j}^{\text {sup }}=\frac{1}{k} \max _{i=1, \ldots, m} \frac{X_{i j}}{i} .
\end{aligned}
$$

Therefore, if $x_{1 j}=\cdots=x_{m j}$ for some $j \in\{1, \ldots, n\}$, then $s_{j}^{\text {inf }}=s_{j}^{\text {sup }}$, in which case the interval $\left(s_{j}^{\text {inf }}, s_{j}^{\text {sup }}\right)$ is empty and we cannot find $s_{j}^{M}$ with $s_{j}^{\text {inf }}<s_{j}^{M}<s_{j}^{\text {sup }}$. Consequently, Khodabakhshi and Aryavash's model can be employed only when $\min _{i=1, \ldots, m} x_{i j} \neq \max _{i=1, \ldots, m} x_{i j}$ for every $j \in\{1, \ldots, n\}$.

When it comes to finding a global score, the authors suppose, without any proof, that there exists $\lambda \in(0,1)$ solution of System (3). Again Theorem 1 allows us to justify the existence of $\lambda \in(0,1)$. Under the assumption that $s_{j}^{\text {inf }}<s_{j}^{\text {sup }}$ for each $j \in\{1, \ldots, n\}$, we get $\sum_{j=1}^{n} s_{j}^{\text {inf }}<\sum_{j=1}^{n} s_{j}^{\text {sup }}$; so $\lambda$ is well defined through expression (5). Moreover, notice that the condition $0<\lambda<1$ is equivalent to $\sum_{j=1}^{n} s_{j}^{\text {inf }}<1<\sum_{j=1}^{n} s_{j}^{\text {sup }}$. Since for any $l \in\{1, \ldots, m\}$ we have $\sum_{j=1}^{n} x_{l j}=k$, then $\sum_{j=1}^{n} X_{l j}=l k$, or, equivalently, $\sum_{j=1}^{n} X_{l j} / l=k$. Therefore,

$$
\sum_{j=1}^{n} s_{j}^{\text {sup }}=\frac{1}{k} \sum_{j=1}^{n} \max _{i=1, \ldots, m} \frac{X_{i j}}{i}>\frac{1}{k} \sum_{j=1}^{n} \frac{X_{l j}}{l}=1,
$$

where $l \in\{1, \ldots, m\}$ is chosen so that $X_{l 1} / l<\max _{i=1, \ldots, m} X_{i 1} / i$. The condition $\sum_{j=1}^{n} s_{j}^{\text {inf }}<1$ can be proven in a similar way.

A comment also deserves the restriction $\sum_{i=1}^{m} w_{i}=1 / k$. According to the authors, this restriction is obtained from the normalization assumption $\sum_{j=1}^{n} s_{j}=1$ as follows:

$$
1=\sum_{j=1}^{n} s_{j}=\sum_{j=1}^{n} \sum_{i=1}^{m} x_{i j} w_{i}=\sum_{i=1}^{m}\left(\sum_{j=1}^{n} x_{i j}\right) w_{i}=\sum_{i=1}^{m} k w_{i}=k \sum_{i=1}^{m} w_{i} .
$$

However, this result is based on the assumption that the weights $w_{i}$ are the same for all the alternatives, which is not true in the model proposed by the authors. Notice that Model (1) is solved for each alternative $A_{j}$, so that the weights obtained for each alternative may be different. Nevertheless, given that

$$
s_{j}^{M}=s_{j}^{\text {inf }} \lambda+s_{j}^{\text {sup }}(1-\lambda)=\frac{1}{k}\left(\lambda \min _{i=1, \ldots, m} \frac{X_{i o}}{i}+(1-\lambda) \max _{i=1, \ldots, m} \frac{X_{i o}}{i}\right),
$$


the constant $k$ (the number of voters) has no influence on the final order of the alternatives. Consequently, the restriction $\sum_{i=1}^{m} w_{i}=1 / k$ could be replaced by $\sum_{i=1}^{m} w_{i}=1$, which is an usual constraint in this context (see, for instance, Contreras et al., 2005).

The last comment on Khodabakhshi and Aryavash's model refers to an important shortcoming, which is also present in other models proposed in the literature (see Llamazares and Peña, 2009): although there is not any change in the number of first, second, .., $m$ th ranks achieved by two alternatives, their relative order may be different due to a variation in the number of first, second, .., $m$ th ranks obtained by other alternatives. This shortcoming is illustrated in Example 1.

Example 1. Table 1 gathers the number of first, second, third and fourth ranks obtained by four alternatives (for instance, two voters place $A_{1}$ in the first position, six voters place it in the second position and so on). Moreover, the sixth column of Table 1 shows the infimum and supremum scores of alternatives whereas the seventh column displays the global scores. As we can see in the eighth column of Table 1, alternative $A_{3}$ is the winner.

Table 1: First set of data, scores and rankings of alternatives.

\begin{tabular}{llllllll}
\hline$A_{j}$ & $x_{1 j}$ & $x_{2 j}$ & $x_{3 j}$ & $x_{4 j}$ & $\left(s_{j}^{\text {inf }}, s_{j}^{\text {sup }}\right)$ & $s_{j}^{M}$ & Ranking \\
\hline$A_{1}$ & 2 & 6 & 7 & 5 & $(0.100,0.250)$ & 0.170 & 4 \\
$A_{2}$ & 4 & 4 & 3 & 9 & $(0.183,0.250)$ & 0.215 & 3 \\
$A_{3}$ & 6 & 9 & 5 & 0 & $(0.250,0.375)$ & 0.308 & 1 \\
$A_{4}$ & 8 & 1 & 5 & 6 & $(0.225,0.400)$ & 0.307 & 2 \\
\hline
\end{tabular}

Now, suppose that alternatives $A_{1}$ and $A_{2}$ swap a first and a third rank without any variation in the ranks of alternatives $A_{3}$ and $A_{4}$. Table 2 collects the new data, the scores and the rankings of alternatives. As we can see in the eighth column of Table 2, alternative $A_{4}$ is the new winner.

On the other hand, Khodabakhshi and Aryavash's model presents another drawback that is even more grave than the previous one: it is not monotonic; that is, a winning alternative may lose when it improves its results. This fact is illustrated in Example 2.

Example 2. Consider again Table 1, where $A_{3}$ is the winner. Suppose now that this alternative wins one first rank (and loses one second rank) from alternative $A_{1}$. The new data, the scores and the ranking of 
Table 2: Second set of data, scores and rankings of alternatives.

\begin{tabular}{llllllll}
\hline$A_{j}$ & $x_{1 j}$ & $x_{2 j}$ & $x_{3 j}$ & $x_{4 j}$ & $\left(s_{j}^{\text {inf }}, s_{j}^{\text {sup }}\right)$ & $s_{j}^{M}$ & Ranking \\
\hline$A_{1}$ & 1 & 6 & 8 & 5 & $(0.050,0.250)$ & 0.153 & 4 \\
$A_{2}$ & 5 & 4 & 2 & 9 & $(0.183,0.250)$ & 0.218 & 3 \\
$A_{3}$ & 6 & 9 & 5 & 0 & $(0.250,0.375)$ & 0.314 & 2 \\
$A_{4}$ & 8 & 1 & 5 & 6 & $(0.225,0.400)$ & 0.315 & 1 \\
\hline
\end{tabular}

alternatives are gathered in Table 3. As we can see in the eighth column of this table, alternative $A_{4}$ is the new winner.

Table 3: Third set of data, scores and rankings of alternatives.

\begin{tabular}{llllllll}
\hline$A_{j}$ & $x_{1 j}$ & $x_{2 j}$ & $x_{3 j}$ & $x_{4 j}$ & $\left(s_{j}^{\text {inf }}, s_{j}^{\text {sup }}\right)$ & $s_{j}^{M}$ & Ranking \\
\hline$A_{1}$ & 1 & 7 & 7 & 5 & $(0.050,0.250)$ & 0.153 & 4 \\
$A_{2}$ & 4 & 4 & 3 & 9 & $(0.183,0.250)$ & 0.218 & 3 \\
$A_{3}$ & 7 & 8 & 5 & 0 & $(0.250,0.375)$ & 0.314 & 2 \\
$A_{4}$ & 8 & 1 & 5 & 6 & $(0.225,0.400)$ & 0.315 & 1 \\
\hline
\end{tabular}

The previous shortcomings are due to the choice of $\lambda$, because changes in the results obtained by some alternative may affect the global score of alternatives whose results do not change. An easy solution to avoid the previous drawback is to fix the value of $\lambda$. Obviously the choice of $\lambda$ determines the overall order of alternatives. For instance, since $s_{j}^{M}=s_{j}^{\text {inf }} \lambda+s_{j}^{\text {sup }}(1-\lambda)$, when $\lambda=0$ the alternatives are ordered according to the values of $s_{j}^{\text {sup }}$, while $s_{j}^{\text {inf }}$ are the values used when $\lambda=1$. Let us consider in more detail the order between two alternatives $A_{p}$ and $A_{q}$. We may assume, without loss of generality, that $s_{p}^{\text {sup }} \geq s_{q}^{\text {sup }}$. We distinguish the following cases:

1. If $s_{p}^{\text {sup }}>s_{q}^{\text {sup }}$ :
(a) If $s_{p}^{\text {inf }}>s_{q}^{\text {inf }}$, then $s_{p}^{M}>s_{q}^{M}$ for all $\lambda \in[0,1]$.
(b) If $s_{p}^{\text {inf }}=s_{q}^{\text {inf }}$, then $s_{p}^{M}>s_{q}^{M}$ for all $\lambda \in[0,1)$, and $s_{p}^{M}=s_{q}^{M}$ when $\lambda=1$.
(c) If $s_{p}^{\text {inf }}<s_{q}^{\text {inf }}$, then $s_{p}^{M}>s_{q}^{M}$ for all $\lambda \in\left[0, \alpha_{p q}\right), s_{p}^{M}=s_{q}^{M}$ when $\lambda=\alpha_{p q}$, and $s_{p}^{M}<s_{q}^{M}$ for all 
$\lambda \in\left(\alpha_{p q}, 1\right]$, where

$$
\alpha_{p q}=\frac{1}{1+\frac{s_{q}^{i n f}-s_{p}^{i n f}}{s_{p}^{u l p}-s_{q}^{s u p}}} .
$$

2. If $s_{p}^{\text {sup }}=s_{q}^{\text {sup }}$ :

(a) If $s_{p}^{\text {inf }}>s_{q}^{\text {inf }}$, then $s_{p}^{M}>s_{q}^{M}$ for all $\lambda \in(0,1]$, and $s_{p}^{M}=s_{q}^{M}$ when $\lambda=0$.

(b) If $s_{p}^{\text {inf }}=s_{q}^{\text {inf }}$, then $s_{p}^{M}=s_{q}^{M}$ for all $\lambda \in[0,1]$.

(c) If $s_{p}^{\text {inf }}<s_{q}^{\text {inf }}$, then $s_{p}^{M}<s_{q}^{M}$ for all $\lambda \in(0,1]$, and $s_{p}^{M}=s_{q}^{M}$ when $\lambda=0$.

Clearly, when $\lambda=1 / 2$, the global score of each alternative is the average of the infimum and supremum scores, which seems a fair overall score. Notice that in the three cases considered in the previous examples the average of the infimum and supremum scores coincides for both alternatives $A_{3}$ and $A_{4}$ $((0.25+0.375) / 2=(0.225+0.4) / 2=0.3125)$, and the tie has to be broken through the lexicographic order of the vectors $\left(x_{1 j}, x_{2 j}, x_{3 j}, x_{4 j}\right)$. In this case, the winner is $A_{4}$.

\section{Concluding remarks}

In this paper we have studied a model proposed by Khodabakhshi and Aryavash for aggregating preference rankings into a single ranking by using variable weights. This model is based on considering both the optimistic and pessimistic approaches, for which they maximize and minimize the scores of alternatives by considering several restrictions on the weights. In our analysis, we have provided closed-form expressions for the minimum and maximum scores obtained by the alternatives. These expressions are of great utility because they allow us to know the score obtained by each alternative without solving the proposed model. Moreover, they also allow us to carry out an analysis of some features of the model. As we have seen in Section 4 , some small modifications may be made to improve model performance.

\section{Acknowledgements}

The author is grateful to two anonymous referees for valuable suggestions and comments.

\section{Funding}

This work is partially supported by the Spanish Ministry of Economy and Competitiveness (Project ECO2016-77900-P). 


\section{References}

Bazaraa, M. S., Jarvis, J. J., \& Sherali, H. D. (2009). Linear Programming and Network Flows. (4th ed.). Wiley.

Chebotarev, P. Y., \& Shamis, E. (1998). Characterizations of scoring methods for preference aggregation. Annals of Operations Research, 80, 299-332.

Contreras, I. (2011). A DEA-inspired procedure for the aggregation of preferences. Expert Systems with Applications, $38,564-70$.

Contreras, I., Hinojosa, M. A., \& Mármol, A. M. (2005). A class of flexible weight indices for ranking alternatives. IMA Journal of Management Mathematics, 16, 71-85.

Cook, W. D., \& Kress, M. (1990). A data envelopment model for aggregating preference rankings. Management Science, 36 , $1302-10$.

Ebrahimnejad, A. (2012). A new approach for ranking of candidates in voting systems. OPSEARCH, 49, 103-15.

Fishburn, P. C. (1974). Paradoxes of voting. American Political Science Review, 68, 537-46.

Fishburn, P. C. (1981). Inverted orders for monotone scoring rules. Discrete Applied Mathematics, 3, 27-36.

Foroughi, A. A., \& Aouni, B. (2012). New approaches for determining a common set of weights for a voting system. International Transactions in Operational Research, 19, 521-30.

Foroughi, A. A., Jones, D. F., \& Tamiz, M. (2005). A selection method for a preferential election. Applied Mathematics and Computation, 163, 107-16.

Foroughi, A. A., \& Tamiz, M. (2005). An effective total ranking model for a ranked voting system. Omega, 33, 491-6.

Green, R. H., Doyle, J. R., \& Cook, W. D. (1996). Preference voting and project ranking using DEA and cross-evaluation. European Journal of Operational Research, 90, 461-72.

Hadi-Vencheh, A. (2014). Two effective total ranking models for preference voting and aggregation. Mathematical Sciences, 8 , $1-4$.

Hashimoto, A. (1997). A ranked voting system using a DEA/AR exclusion model: A note. European Journal of Operational Research, 97, 600-4.

Hashimoto, A., \& Wu, D.-A. (2004). A DEA-compromise programming model for comprehensive ranking. Journal of the Operations Research Society of Japan, 47, 73-81.

Hosseinzadeh Lotfi, F., Rostamy-Malkhalifeh, M., Aghayi, N., Ghelej Beigi, Z., \& Gholami, K. (2013). An improved method for ranking alternatives in multiple criteria decision analysis. Applied Mathematical Modelling, 37, 25-33.

Khodabakhshi, M., \& Aryavash, K. (2015). Aggregating preference rankings using an optimistic-pessimistic approach. Computers $\mathcal{E}$ Industrial Engineering, 85, 13-6.

Llamazares, B. (2016). Ranking candidates through convex sequences of variable weights. Group Decision and Negotiation, 25, 567-84.

Llamazares, B., \& Peña, T. (2009). Preference aggregation and DEA: An analysis of the methods proposed to discriminate efficient candidates. European Journal of Operational Research, 197, 714-21.

Llamazares, B., \& Peña, T. (2013). Aggregating preferences rankings with variable weights. European Journal of Operational Research, 230, 348-55.

Llamazares, B., \& Peña, T. (2015a). Positional voting systems generated by cumulative standings functions. Group Decision and Negotiation, 24, 777-801. 
Llamazares, B., \& Peña, T. (2015b). Scoring rules and social choice properties: some characterizations. Theory and Decision, 78 , 429-50.

Merlin, V. (2003). The axiomatic characterizations of majority voting and scoring rules. Mathématiques et Sciences Humaines, 163, 87-109.

Moulin, H. (1988). Axioms of Cooperative Decision Making volume 15 of Econometric Society Monographs. Cambridge: Cambridge University Press.

Obata, T., \& Ishii, H. (2003). A method for discriminating efficient candidates with ranked voting data. European Journal of Operational Research, 151, 233-7.

Stein, W. E., Mizzi, P. J., \& Pfaffenberger, R. C. (1994). A stochastic dominance analysis of ranked voting systems with scoring. European Journal of Operational Research, 74, 78-85.

Wang, N. S., Yi, R. H., \& Liu, D. (2008). A solution method to the problem proposed by Wang in voting systems. Journal of Computational and Applied Mathematics, 221, 106-13.

Wang, Y. M., \& Chin, K. S. (2007). Discriminating DEA efficient candidates by considering their least relative total scores. Journal of Computational and Applied Mathematics, 206, 209-15.

Wang, Y. M., Chin, K. S., \& Yang, J. B. (2007a). Three new models for preference voting and aggregation. Journal of the Operational Research Society, 58, 1389-93.

Wang, Y. M., Luo, Y., \& Hua, Z. (2007b). Aggregating preference rankings using OWA operator weights. Information Sciences, 177, 3356-63. 